\title{
Spike sorting based on PCA and improved fuzzy c-means
}

\author{
Yuyi $^{1, ~ a, ~ Z h a o y u n ~}{ }^{2, b}$, Liuhan ${ }^{3, c}$,Dong Bingchao ${ }^{4, d}$, Li Zhenxin ${ }^{5, e^{*}}$ \\ ${ }^{1}$ No. 601 Jinsui Road, Xinxiang 453003, Xinxiang Medical University, Henan, China \\ ${ }^{2}$ No. 601 Jinsui Road, Xinxiang 453003, Xinxiang Medical University, Henan, China \\ ${ }^{3}$ No. 601 Jinsui Road, Xinxiang 453003, Xinxiang Medical University, Henan, China \\ ${ }^{4}$ No. 601 Jinsui Road, Xinxiang 453003, Xinxiang Medical University, Henan, China \\ ${ }^{5}$ No. 601 Jinsui Road, Xinxiang 453003, Xinxiang Medical University, Henan, China \\ ayuyi@xxmu.edu.cn, byyy90@163.com, cliuhan0234@163.com, dbchdong@163.com, \\ 'lizhenxin200801@163.com
}

Keywords: Spike sorting; detection; clustering; PCA; improvement fuzzy c-means

Abstract: Proper classification of spikes from extracellular recordings is essential for the study of neuronal behavior. A lot of algorithms have been presented in the technical literature. Combining with subtractive clustering and fuzzy c-means, we present a new algorithm named improved fuzzy c-means. Compared with fuzzy c-means, the dependence on the initial centers of improved fuzzy c-means is reduced. Not only do the new algorithm improve the accuracy of classification, but also the results of classification are more stable. Three types of classifier were employed in this paper to assess the performance of the spike sorting algorithm. When the noise level rises gradually, the accuracy of k-means of fuzzy c-means decreased quickly.

\section{Introduction}

Neural interface has been one of the most amazing technologies in bioengineering and neuroscience because it provides an alternate communication channel between neural system and man-made devices[1]. Neural action potentials, the so-called spikes, play a key role in the neural interfaces. Neurons in brain transmit messages by spikes, so spike sorting is the first and a critical step $[2,3,4]$.

Most spike sorting algorithms employ three principal steps: detecting spikes from the recorded data, extracting features characteristic of the spikes and clustering the spikes of individual neurons based on the extracted features[5,6]. The first step includes amplitude thresholding[7,8] and a nonlinear energy operator[9]. In the feature extraction step, there are principal components analysis(PCA)[10] and wavelet basis[11]. The clustering step contains too many methods, such as bayes methods, k-means, hierarchical clustering, fuzzy c-means, and a variety of artificial neural network. $[12,13]$ use bayes model for clustering, which similarly has a high accuracy, however, can't deal with large samples, besides, signals are affected by background noise greatly. In the recent years, with the development of artificial neural networks, [14] classifies the low ratio signals via artificial neural networks whereas the accuracy is not very well. Fuzzy c-means and k-means do a good job in $[15,16]$. These clustering algorithms are based on distance, nevertheless, their initial cluster centers are generated randomly. It might be influenced greatly by the location of the initial cluster centers.

The fuzzy clustering algorithm establishes uncertain description of samples on the categories which must be better to reflect the objective world. This paper presents an improved fuzzy c-means. We use subtractive clustering to determine the number of categories and the location of the cluster centers and then utilize the fuzzy c-means for classification. 


\section{Methods}

Spikes are detected by the nonlinear energy operator. In the feature extraction, the detected spikes were transformed into PCA displaying distributions with multiple peaks were selected for spike clustering. Finally, the data were clustered into groups of spikes belonging to different neurons.

\section{Spike detection}

The spike detection employed in this paper is base on nonlinear energy operator. The signals were recorded at the sampling frequency of $30 \mathrm{KHz}$. First, they must undergo a band-pass filter aimed at removing slowly changing local field potential(LFP) and high-frequency fluctuations. Here we use the typical FIR band-pass filter. The band-pass range and order of the filter are $800 \mathrm{~Hz}-4 \mathrm{kHz}$ and 60 , respectively. After the band-pass filtering, we use the time window to process the signals:

$$
x_{\mathrm{N}}(n)=\omega_{R}(n) x(n)=\left\{\begin{array}{cl}
x(n), & 0 \leq n \leq N-1 \\
0, & \text { else }
\end{array}\right.
$$

Where $x(n)$ refers to the sampled filtered signal, and $\omega_{\mathrm{R}}(n)$ in Eq. 1 is defined as

$$
c_{\mathrm{B}}(n)=\left\{\begin{array}{c}
1,0 \leq n \leq \mathrm{N}-1 \\
0, \text { else }
\end{array}\right.
$$

Here the length of the window we choosed is $5 \mathrm{~ms}$. Then we calculate the power of the signals in the window.

Based on the theorem of Wiener-Khinchin, the signal power spectrum can be obtained by the Fourier transform of the autocorrelation function. $\sigma$ is set to be the whole signal energy standard deviation, with 5 times its value $(5 \sigma)$ as the judgment standard of action potential. If the root-mean-square of the signal power spectrum in the window is bigger than $5 \sigma$, the waveform detected within the window was regarded as a spike.

\section{Feature extraction}

At present, principal component analysis(PCA) is a dimension reduction technique which is widely used in the signal processing at present. The dimension reduction procedure of PCA is shown as follows: first, we should calculate the covariance matrix of the spike vector to get the eigenvalues and eigenvectors. Then we sort the eigenvalues in descending order and sort the eigenvectors in the same sequence. According to the eigenvectors we can get a new matrix. At last, we consider the signal vectors with contribution above $85 \%$ as principal component.

\section{clustering}

Standard FCM: The clustering criterion function of fuzzy c-means is

$$
\mathrm{J}_{\mathrm{m}}=\sum_{\mathrm{i}=1}^{\mathrm{c}} \Sigma_{j=1}^{\mathrm{N}}\left(\mu_{\mathrm{ji}}\right)^{\mathrm{m}}\left\|x^{\mathrm{j}}-w_{\mathrm{i}}\right\|^{2}
$$

Where $\left\{x^{\mathrm{j}}, \mathrm{j}=1,2, \ldots, \mathrm{n}\right\}$ is the sample collection consisted of $\mathrm{n}$ samples, $\mathrm{c}$ is defined as categories, $w_{\mathrm{j}}, \mathrm{i}=1,2,3_{1} \ldots, \mathrm{c}$ refers to every cluster center. $\mu_{\mathrm{ji}}$ is the jth samples' membership function for class $\mathrm{i}$, and $\mu_{\mathrm{ji}}$ should satisfy $\sum_{\mathrm{i}=1}^{\mathrm{c}} \mu_{\mathrm{ji}}=1, \sum_{\mathrm{j}=1}^{\mathrm{N}} \mu_{\mathrm{ji}}>0$; the value of $\mathrm{m}$ is usually 2 .

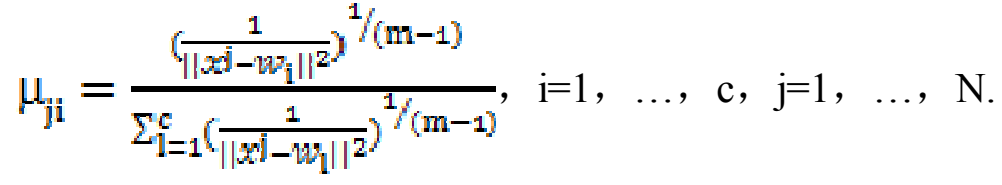

$$
\begin{aligned}
& \left.w_{i}=\sum_{j=1}^{n}\left(\mu_{j i}\right)^{m} X^{3}\right) / \Sigma_{j=1}^{n}\left(\mu_{j i}\right)^{m}
\end{aligned}
$$

The steps of the algorithm are, setting the values of $\mathrm{c}$ and $\mathrm{m}$; initializing every cluster center $w_{1}, 1=1,2, \ldots, c$; repeating the following steps till the membership of every sample is stable: Calculate the membership function with Eq. 4; Update every cluster center with Eq. 5.

Improvement fuzzy c-means: The first two steps of the improvement fuzzy c-means are, setting the value of $m$ and the subtractive clustering parameters, call the subtractive clustering algorithm to get 
initial clustering centers; assigning the clustering centers to FCM, namely $\mathrm{c}=\mathrm{m}, w_{\mathrm{i}}(1) \approx \varphi_{i}, \mathrm{i}=1,2, \ldots$, c. The following steps are the same as the standard FCM.

\section{Results}

Simulated data used in this study were obtained from 128-channel analog signal generator. The sampling frequency is $30 \mathrm{kHz}$. This paper consider the simulated data as raw signal. We detected the spikes by nonlinear energy operator and amplitude thresholding. The adding noise is white Gaussian noise.

Table 1 results of two spike detection algorithm

\begin{tabular}{|c|c|c|c|c|}
\hline \multirow[t]{2}{*}{$\mathrm{SNR}(\mathrm{dB})$} & \multicolumn{2}{|c|}{$\begin{array}{l}\text { Amplitude } \\
\text { thresholding }\end{array}$} & \multirow{2}{*}{$\begin{array}{c}\begin{array}{c}\text { Nonlinear } \\
\text { operator }\end{array} \\
\begin{array}{c}\text { Total } \\
\text { detection }\end{array}\end{array}$} & \multirow{2}{*}{$\begin{array}{l}\text { energy } \\
\text { Effective } \\
\text { number }\end{array}$} \\
\hline & $\begin{array}{c}\text { Total } \\
\text { detection }\end{array}$ & $\begin{array}{l}\text { Effective } \\
\text { number }\end{array}$ & & \\
\hline-10 & 226 & 226 & 226 & 226 \\
\hline-15 & 220 & 220 & 226 & 225 \\
\hline-20 & 167 & 167 & 225 & 225 \\
\hline-25 & 98 & 98 & 229 & 217 \\
\hline-30 & 21 & 21 & 192 & 103 \\
\hline
\end{tabular}

Table 1 compares the two spike detection algorithm with different SNR. In low SNR, the performance of nonlinear energy operator is much better and the anti-noise ability is stronger.

After the detection, we employ PCA to process the signals. We choose the first and second principal component and show the scatter diagram in Fig. 1.

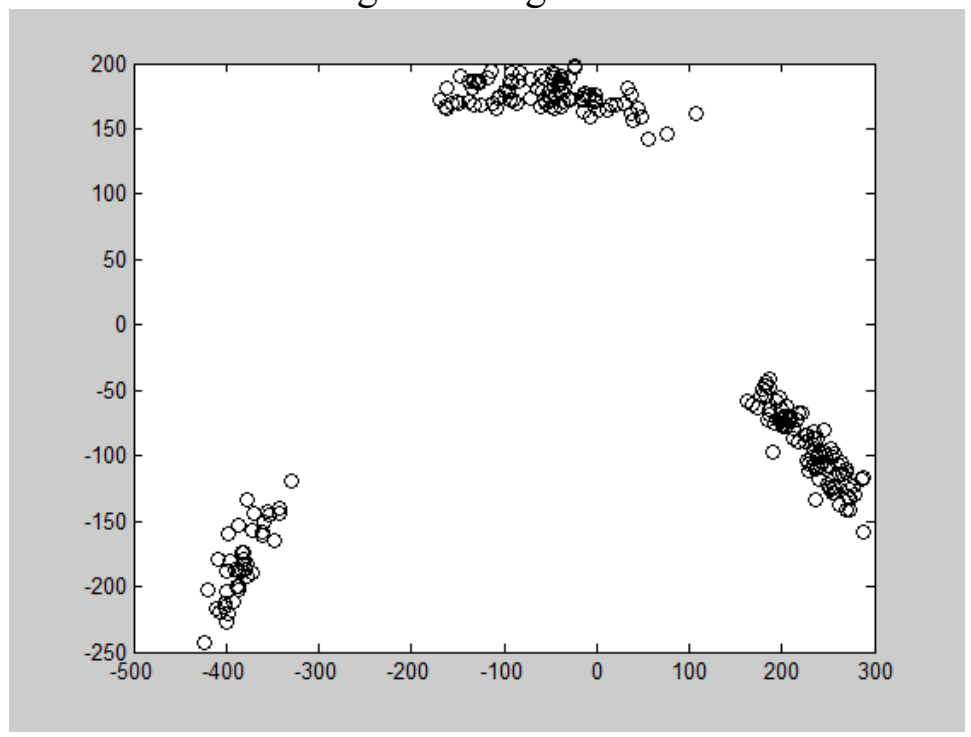

Fig. 1 Scatter diagram of the first and second principal component of spikes

From the scatter diagram, we can see the spikes can be divided into three different categories. After the process of improved fuzzy c-means, the results are shown in Fig. 2. 


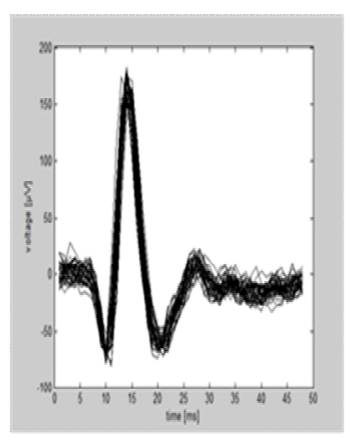

Class A

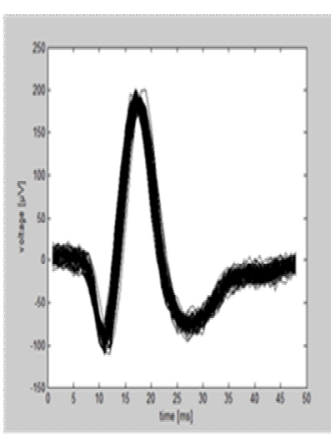

Class B

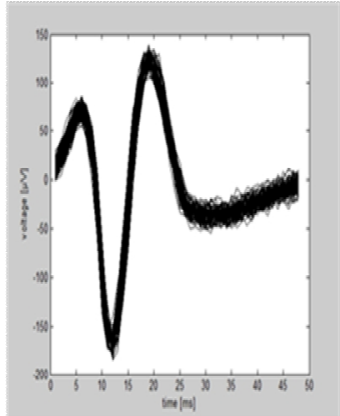

Class C

Fig. 2 Results of clustering

The accuracy of the three clustering algorithms are depicted in Table 2.

Table 2 results of three clustering algorithm

\begin{tabular}{llll}
\hline $\begin{array}{l}\mathrm{SN} \\
\mathrm{R}\end{array}$ & K-means & Standard FCM & Improved FCM \\
\hline- & 100.0 & 100.0 & 100.0 \\
-10 & 99.6 & 100.0 & 100.0 \\
-20 & 99.1 & 99.6 & 100.0 \\
-30 & 98.2 & 98.2 & 100.0 \\
-40 & 92.9 & 93.4 & 96.0 \\
-42 & 86.7 & 90.7 & 93.8 \\
-43 & 77.4 & 81.0 & 89.4 \\
-45 & 66.4 & 71.7 & 84.1 \\
\hline
\end{tabular}

Compared with the accuracy of improved fuzzy c-means when SNR is lower than $-40 \mathrm{~dB}$, the accuracy of k-means and standard FCM falls faster. When SNR is $-10 \mathrm{~dB},-20 \mathrm{~dB},-30 \mathrm{~dB},-40 \mathrm{~dB}$, the accuracy of three algorithm is very close. The initial clustering centers lead to this phenomenon. The initial clustering centers of k-means and standard fuzzy c-means are generated randomly, so the characteristic of samples can't be reflected fully. Thus, it is easy to make the results fall into local optimal. On the whole, the accuracy of improved fuzzy c-means is significantly higher than others.

\section{Discussion}

This paper attempts to implement spike detection through amplitude thresholding and nonlinear energy operator. It is shown that when the SNR is greater than -10, the accuracy of the two algorithms are $100 \%$. With the SNR decreased, the accuracy of amplitude thresholding declined sharply. However, the performance of nonlinear energy operator is very good. Compared with amplitude thresholding, the detection rate and accuracy of nonlinear energy operator still remains high when SNR is -25. Generally, nonlinear energy operator can extract the spikes effectively in the noise background.Combined with subtractive clustering, this paper improves standard FCM. As the initial clustering centers are 
generated by subtractive clustering, the convergence speed of the algorithm also increases. Meanwhile, it also reduces the user's blindness to determine the numbers of clustering.

\section{Acknowledgment}

This study has received kind and generous support from the National Natural Science Foundation of China (61305147) and the Natural Science Foundation of Henan Province (13A416858).

\section{References}

[1] Sanchez Justin C, Principe Jose C .Brain-machine interface engineering. San Francisco: Morgan \& Claypool Publishers, 2007, pp. 1-20.

[2] Lebedev M A, Nicolelis M A L. Brain-machine interface: pase, present and future. Trends in Neurosciences, 2006, 29(9): 536-546.

[3] Becedas, J. Brain-Machine Interfaces: Basis and Advances. Systems, Man, and Cybernetics, Part C: Applications and Reviews, IEEE Transactions on, 2012, 42(6): 825-836.

[4] Miguel Pais-Vieira, Mikhail Lebedev, Carolina Kunicki, Jing Wang, Miguel A.L, Nicolelis. A Brain-to-Brain Interface for Real-Time Sharing of Sensorimotor Information. Sci Rep, 2013, $3: 1319$

[5] Wild J, Prekopcsak Z, Sieger T, Novak D, Jech R. Performance comparison of extracellular spike sorting algorithms for single-channel recordings. J Neurosci Methods, 2012, 203(2): 369-376.

[6] Wu Wei, Gao Yun, Bienenstock Elie, Donoghue John P, Black Michael J. Bayesian Population Decoding of Motor Cortical Activity Using a Kalman Filter. Neural Computation, 2005, 18(1): 80-118.

[7] Takashi Takekawa, Yoshikazu Isomura, Tomoki Fukai. Accurate spike sorting for multi-unit recordings. European Journal of Neuroscience, 2010, 31(2): 263-272.

[8] Mukhopadhyay S, Ray GC. A new interpretation of nonlinear energy operator and its efficacy in spike detection. IEEE Transactions on Biomedical Engineering, 1998, 45(2): 180-187.

[9] Yang Z, Zhao Q, Liu W. Improving spike separation using waveform derivatives. Journal of neural engineering, 2009, 6(4): 1-12.

[10]Quiroga RQ, NAdasdy Z, Ben-Shaul Y. Unsupervised spike detection and sorting with wavelets and superparamagnetic clustering. Neural Computation, 2004, 16(8): 1661-1687.

[11] Sunghan Kim, James McNames. Automatic spike detection based on adaptive template matching for extracellular neural recordings. Journal of Neuroscience Methods, 2007, 165(2): 165-174.

[12]Zhe Chen. An Overview of Bayesian Methods for Neural Spike Train Analysis. Computational Intelligence and Neuroscience, 2013: 1-17.

[13] Takekawa T, Isomura Y,Fukai T. Spike sorting of heterogeneous neuron types by multimodality-weighted PCA and explicit robust variational Bayes. Front Neuroinform,2012, 6(5): 1-13.

[14] Stitt J.P, Gaumond R.P, Frazier J.L, Hanson F.E. An artificial neural network for neural spike classification. Bioengineering Conference, 1997, Proceedings of the IEEE 1997 23rd Northeast, 1997.

[15]Chah E, Hok V, Della-Chiesa A, Miller JJ, O'Mara SM, Reilly RB. Automated spike sorting algorithm based on Laplacian eigenmaps and k-means clustering. Journal of Neural Engineering, 2011, 8(1):1-9.

[16]Inan ZH, Kuntalp M. A study on fuzzy C-means clustering-based systems in automatic spike detection. Computers in Biology and Medicine, 2007, 37(8): 1160-1166. 